\title{
Assortative and modular networks are shaped by adaptive synchronization processes
}

\author{
Vanesa Avalos-Gaytán, ${ }^{1}$ Juan A. Almendral, ${ }^{2,3}$ David Papo, ${ }^{4}$ Satu Elisa Schaeffer, ${ }^{5}$ and Stefano Boccaletti ${ }^{4}$ \\ ${ }^{1}$ Postgraduate Division in Systems Engineering, Department of Mechanical and Electrical Engineering, \\ Universidad Autónoma de Nuevo León, San Nicolás de los Garza, Nuevo León, Mexico \\ ${ }^{2}$ Complex Systems Group, Universidad Rey Juan Carlos, Calle Tulipán s/n, E-28933 Móstoles, Spain \\ ${ }^{3}$ Laboratory of Biological Networks, Center for Biomedical Technology, E-28223 Pozuelo de Alarcón, Madrid, Spain \\ ${ }^{4}$ Group of Computational Systems Biology, Center for Biomedical Technology, E-28223 Pozuelo de Alarcon, Madrid, Spain \\ ${ }^{5}$ Postgraduate Division in Computation and Mechatronics, Department of Mechanical and Electrical Engineering, \\ Universidad Autónoma de Nuevo León, San Nicolás de los Garza, Nuevo León, Mexico \\ (Received 26 March 2012; revised manuscript received 9 May 2012; published 26 July 2012)
}

\begin{abstract}
Modular organization and degree-degree correlations are ubiquitous in the connectivity structure of biological, technological, and social interacting systems. So far most studies have concentrated on unveiling both features in real world networks, but a model that succeeds in generating them simultaneously is needed. We consider a network of interacting phase oscillators, and an adaptation mechanism for the coupling that promotes the connection strengths between those elements that are dynamically correlated. We show that, under these circumstances, the dynamical organization of the oscillators shapes the topology of the graph in such a way that modularity and assortativity features emerge spontaneously and simultaneously. In turn, we prove that such an emergent structure is associated with an asymptotic arrangement of the collective dynamical state of the network into cluster synchronization.
\end{abstract}

DOI: 10.1103/PhysRevE.86.015101

PACS number(s): 89.75.Hc, 05.45.Xt

Natural networking systems [1] are vastly characterized by a modular organization of their connectivity structure [2], and by nontrivial correlation features in the way units with a given number of connections (degree) tend to link with members of the same degree (assortativity), or with units with different degrees (disassortativity). Modularity is clearly the result of the need for social, biological, and technological systems to optimize their parallel, yet integrated, functioning [3,4] by means of an organization into mesoscale structures, such as communities, i.e., groups of highly interconnected nodes that are sparsely connected to the rest of the graph [5]. Degreedegree correlation reflects the observed tendency of natural networks to organize the main topology on top of a backbone of nodes that may be starlike (disassortativity) or of highly connected hubs (assortativity).

Whereas the emphasis typically has been on devising suitable tools and algorithms to unveil, identify, and quantify both modular and correlation features in real world networks [1,2], a model that has proven to be capable of generating these features simultaneously is needed. Modularity and assortativity are usually imposed separately, in a second step, on top of already generated networks by means of ad hoc algorithms and methods [6,7].

In this Rapid Communication, we show that all these features may spontaneously emerge in an adaptive network of interacting oscillators as the result of a delicate interplay between synchronization processes and coevolution of the connectivity structure. When the connectivity dynamics is such that links coupling the nodes with synchronous (nonsynchronous) dynamics are promoted (weakened), we prove that an initially unstructured clique configuration evolves in time toward an emerging structured network displaying both modularity and assortativity.

Let us then start by considering an initial ensemble of $N$ all-to-all coupled Kuramoto oscillators [8,9]. Each unit of the ensemble $n=1, \ldots, N$ is characterized by its phase $\theta_{n}$, whose dynamics is ruled by

$$
\dot{\theta}_{n}=\Omega_{n}+\frac{\sigma}{N} \sum_{m=1}^{N} w_{n m} \sin \left(\theta_{m}-\theta_{n}\right),
$$

where $\Omega_{n}$ is the natural frequency of the $n$th oscillator (taken at random from a uniform distribution in the interval $[0.8,1.2]$ ), $w_{n m} \in[0,1]$ is the weight of the connection between the units $n$ and $m$, and $\sigma$ is the coupling strengt $h$, which here acts as a first global parameter.

In turn, we suppose that the connection weights coevolve with the dynamics of the units. Namely, they are taken to be time dependent variables $\left[w_{n m}=w_{n m}(t)\right]$ that obey the following equation:

$$
\dot{w}_{n m}=\left(p_{n m}-p_{c}\right) w_{n m}\left(1-w_{n m}\right),
$$

where $p_{n m}$ is the instantaneous phase correlation between units $n$ and $m$, resulting from

$$
p_{n m}(t):=\frac{1}{2}\left|e^{i \theta_{n}(t)}+e^{i \theta_{m}(t)}\right|,
$$

and $p_{c}$ (the correlation threshold) is the second parameter of the model.

It is worth noticing that, from Eq. (3), $p_{n m}$ is exactly one for all pairs of units with equal phases, while it vanishes for pairs of oscillators with opposite phases, $\theta_{n}=\theta_{m} \pm \pi$. The parameter $p_{c}$ has the following meaning: A link weight is reinforced at all times at which $p_{r m}>p_{c}$, whereas it weakens when $p_{n m}(t)<p_{c}$. Thus, connections improving (reducing) the degree of synchronization between a pair of oscillators are reinforced (weakened). The driving force for the weight dynamics [the right-hand side of Eq. (2)] has two attractors, leading each weight to asymptotically converge to either one of the values in $\{0,1\}$.

As a consequence, for any given choice of $\sigma$ and $p_{c}$, a generic random initial condition for all $\theta_{n}(0)$ and for all $w_{n m}(0)$ results in a progressive pruning of the units' connections, up to 
when, ultimately, Eqs. (1) and (2) stick into an asymptotic state (defined as $\dot{w}_{n m}=0, \forall n, m$ ), which corresponds to a specific dynamical organization of the ensemble, and to the spontaneous emergence of a given, unweighted, network topology.

Attainment of the asymptotic state is numerically verified by checking either $w_{n m}(t)>1-\epsilon$ or $w_{n m}(t)<\epsilon$ from a given time on (in all our trials $\epsilon=10^{-3}$ ), ensuring that the original all-to-all weighted connectivity matrix is sufficiently close to a network adjacency matrix. We further require $\dot{w}_{n m}<\epsilon$ to check the stability of the convergence process. Upon reaching a link configuration fulfilling the above conditions, we proceed to round each $w_{n m}$ to its nearest integer value. Numerical evidence shows that the model always fulfills the stopping criterion, though the convergence time crucially depends on the specific values of $p_{c}$ and $\sigma$ (being, in only a few cases, one or two orders of magnitude longer than a characteristic time scale of the order of 1000 cycles of the oscillator with lower natural frequency).

It is also crucial to stress that here the phase correlation [Eq. (3)] is an instantaneous measure, not depending on any long-term synchronization processes. This constitutes a qualitative difference between the present study and the one conducted in Ref. [10], where it was shown that memory dependent adaptation mechanisms may induce clustering synchronization and the simultaneous appearance of a scalefree distribution for the weights of a network. Here, instead, the adaptive nature of the interactions directly shapes the topology of a network, and below we will only be discussing the connectivity properties of the emergent network structure.

As for the asymptotic dynamics, the degree of global synchronization in the Kuramoto model is traditionally mea- sured in $[0,1]$ by means of the order parameter $R^{G}:=$ $\left\langle\frac{1}{N}\left|\sum_{n=1}^{N} e^{i \theta_{n}(t)}\right|\right\rangle_{t}$ (with $R^{G} \approx 1$ indicating a fully synchronized graph, and $R^{G} \approx 0$ corresponding to an asynchronous behavior). However, relevant situations may occur in which the network is globally unsynchronized and yet groups of nodes in it display a high level of local synchrony. To measure the degree of local synchronization around node $n$, here we consider

$$
R_{n}^{L}:=\left\langle\left|\frac{\sum_{m=1}^{N} w_{n m} e^{i \theta_{m}(t)}}{\sum_{m=1}^{N} w_{n m}}\right|\right\rangle_{t}
$$

and, consequently, the average degree of local synchronization over the whole network is

$$
R^{L}:=\frac{1}{N} \sum_{n=1}^{N} R_{n}^{L} .
$$

In Fig. 1 we report $R^{G}$ [Fig. 1(a)] and $R^{L}$ [Fig. 1(b)] vs $p_{c}$, characterizing the asymptotic dynamics of the network for different values of $\sigma$. Each point in it, as well as in the rest of the figures, is the ensemble average of 100 independent realizations, each one starting from different initial conditions. At low values of $p_{c}$, a clear transition from a local and global unsynchronized to a synchronized behavior is observed at $\sigma \approx 0.26$ [see Fig. 1(d)]. Such a critical coupling strength very much coincides with that characterizing the original all-to-all Kuramoto model [11], $\sigma_{c}=0.8 / \pi \simeq 0.2547$. On the contrary, the range $p_{c}>0.6$ is characterized by a decreasing degree of global order, associated, however, with an initially decreasing and lately increasing degree of local synchronization. This is evidently marking the raising of cluster synchronization,
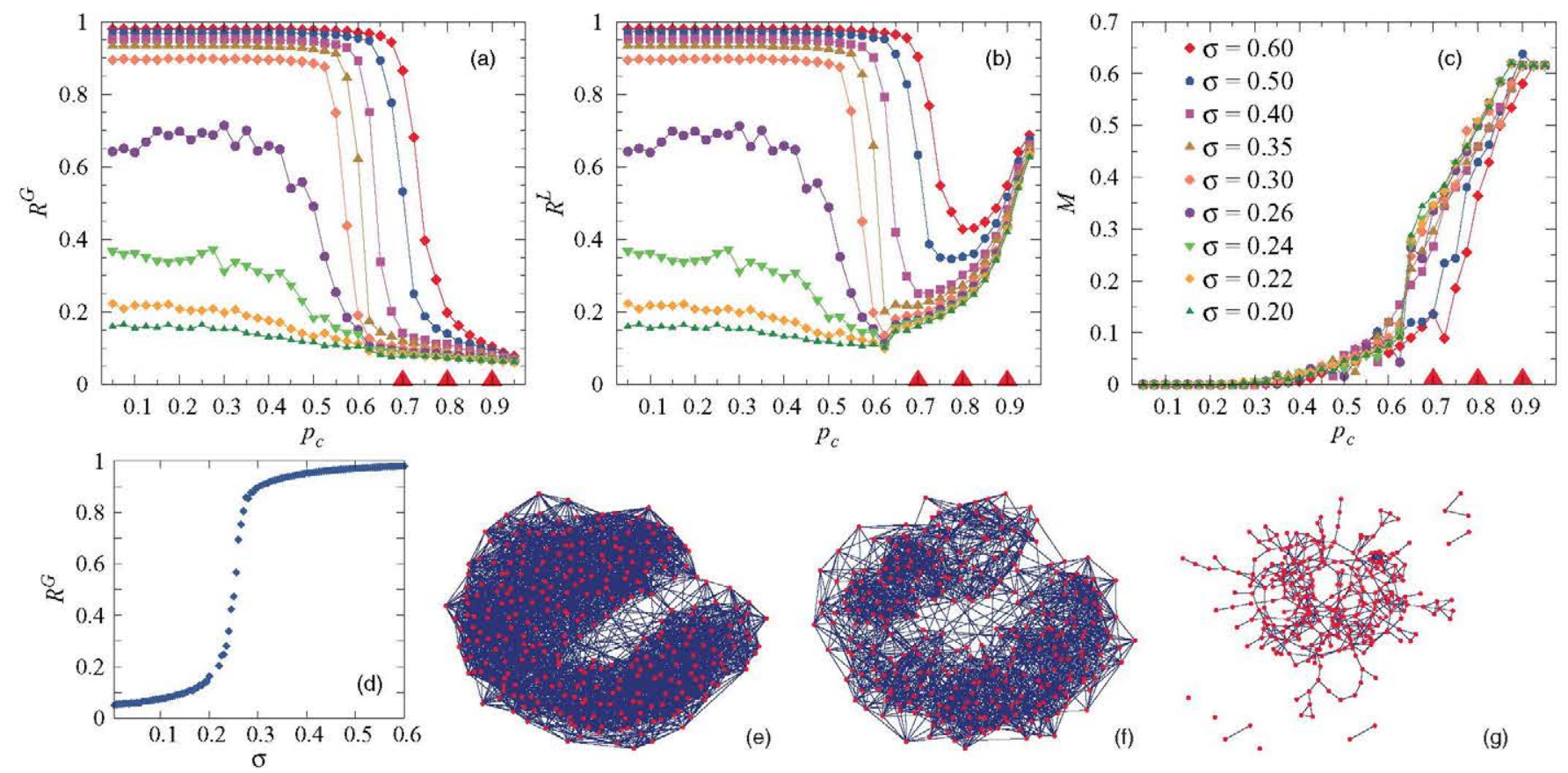

FIG. 1. (Color online) Top: (a) Global synchronization indicator $R^{G}$ (see text for definition), (b) local synchronization indicator, from Eq. (5), and (c) modularity index $\mathcal{M}$, from Eq. (6), vs $p_{c}$. Each curve corresponds to a different value of the coupling strength $\sigma$, represented with the symbols and colors shown in the legend of (c). Bottom: (d) Transition to synchronization in the model, using the coupling strength $\sigma$ as the order parameter, for $p_{c}=0.25$, and typical network configurations obtained for (e) $p_{c}=0.7$, (f) $p_{c}=0.8$, and (g) $p_{c}=0.9$ (in all cases $\sigma=0.2$ ). The three specific values of the correlation threshold are marked with red upward pointing arrows in all the three top panels. 
where oscillators form communities of given frequencies (and then there is a high level of local synchronization), which, however, are unsynchronized between them.

One of the major results of our study is that, as a consequence of the adaptive evolution of the ensemble, specific mesoscales are shaped in the final topology. To properly visualize the presence of communities, we measure the modularity index [12] of the network in its asymptotic state, defined by

$$
\mathcal{M}:=\frac{1}{2 M} \sum_{m, n}\left(a_{m n}-\frac{k_{m} k_{n}}{2 M}\right) \delta\left(c_{m}, c_{n}\right) .
$$

In this expression $a_{m n}$ is the asymptotic value of the weight $w_{m n}$ (i.e., 0 or 1 ), the index $c_{n}$ refers to the cluster which the node $n$ belongs to, $k_{n}$ is the degree of the $n$th node, $\delta$ is the Kronecker delta function, and $M$ is the total number of links in the graph. The clusters $c_{n}$ in the community structure are obtained by means of the fast algorithm described in Ref. [13].

The results are shown in Fig. 1(c). The remarkable result is that there is an absence of modularity for all those values of $p_{c}$ where global and local synchronization values are high, whereas $\mathcal{M}$ exhibits a rapid transition where $p_{c}$ is around $0.6-0.7$, i.e., for low global, but high local, values of the synchronization indicators.

While a transition to a modular structure is a generic feature, the specific transition value of $p_{c}$ depends instead on the type of initial distribution of the natural frequencies. In particular, for Gaussian distributions, we found wider ranges for the transition point around $p_{c} \sim 0.6-0.8$, while for a double-peaked distribution, the transition range is rather narrow, close to $p_{c}=0.6$.

A second inspected structural feature of the emerging network is represented by assortativity, i.e., the correlation features of the degrees of neighboring nodes. For this purpose, we measure the assortativity coefficient $r$ [14], which is essentially the Pearson correlation coefficient of the degree between pairs of linked nodes, and it is given by

$$
r:=\frac{1}{\sigma_{q}^{2}} \sum_{n, m} n m\left(e_{n m}-q_{n} q_{m}\right) .
$$

$q_{n}$ is the distribution of the remaining degree, and it can be obtained from the degree distribution $p_{n}$ as $q_{n}:=\frac{(n+1) p_{n+1}}{\sum_{m} m p_{m}} \cdot e_{n m}$ is the joint probability distribution of the remaining degrees of the two vertices, and $\sigma_{q}^{2}$ is the variance of the distribution $q_{n}$. The assortativity coefficient $r$ always lies in $[-1,1]$, and if $r>0$, the network is said to be assortative.

An alternative way to quantify the degree correlation is to compute the average degree of the neighbors of a node with given degree $k$ [15],

$$
k_{n n}(k):=\sum_{k^{\prime}} k^{\prime} P\left(k^{\prime} \mid k\right),
$$

where $P\left(k^{\prime} \mid k\right)$ is the conditional probability for a node with degree $k$ to have a neighbor with degree $k^{\prime}$. If $k_{n n}(k)$ is a monotonically increasing function, then the network is assortative.

In Fig. 2 we report the assortativity coefficient $r$ vs $p_{c}$, for different values of $\sigma$ [with the same color and symbol stipulations used for the curves in Figs. 1(a)-1(c)].

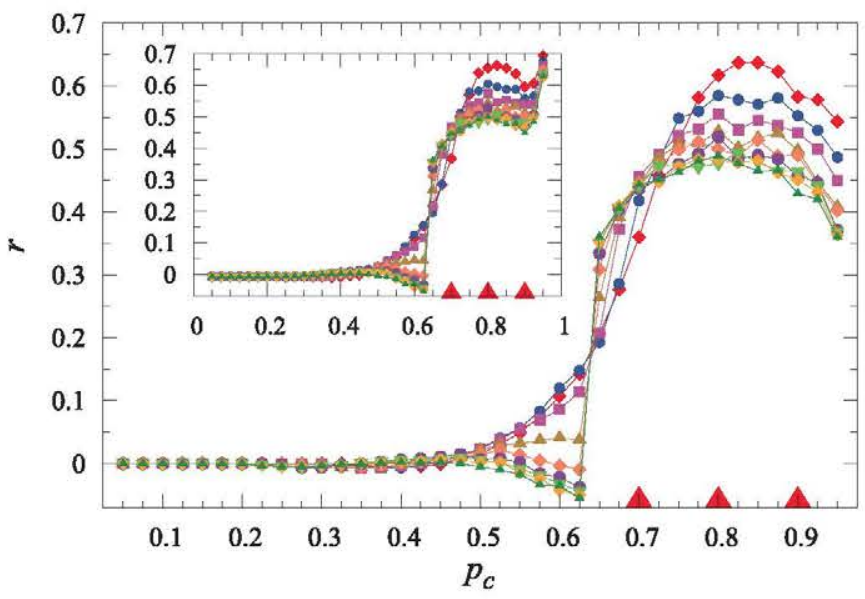

FIG. 2. (Color online) Assortativity coefficient $r$ [as defined in Eq. (7)] vs $p_{c}$, for different values of $\sigma$ (same stipulations for colors and symbols of the curves as in the caption of Fig. 1). The plot in the inset depicts the slope of the best linear fit of the function $k_{n n}(k)$ vs $p_{c}$. Notice that both plots clearly indicate a transition to an assortative configuration for $p_{c}>0.6$, i.e., in correspondence with the transition to increasingly pronounced modular networks highlighted in Fig. 1(c).

Furthermore, the plot in the inset shows the slope of the best linear fit of the function $k_{n n}(k)$ (in the vertical axis) versus $p_{c}$ (in the horizontal axis). Remarkably, for $p_{c}>0.6$, in perfect correspondence with the transition to increasingly pronounced modular structures already observed in Fig. 1(c), here both plots also indicate a transition to an assortative configuration of the network in its final state. This latter transition (from a nonassortative to an assortative topology) is moreover rather independent of the value of $\sigma$, and is always observed in the range $p_{c}=0.6-0.7$.

The last result that is worth mentioning is about the long transient behaviors observed in those cases, where the time needed to attain the final network structure is very long. This transient behavior is essentially due to the persistent frustration of a few links in the network (typically less than 100). The results of Ref. [16] indicate that, indeed, in networks consisting of two modules of interacting phase oscillators, most oscillators are locked to the synchronous behavior of one of the modules, but a few oscillators are in a frustrated situation as they receive inputs from nodes belonging to different clusters. As a consequence, if nodes in the core of the two modules have frequencies $\Omega_{1}$ and $\Omega_{2}$, the instantaneous frequency of the frustrated nodes is time dependent, and oscillates around the value $\left(\Omega_{1}+\Omega_{2}\right) / 2$ with frequency $\left(\Omega_{1}-\Omega_{2}\right) / 2$.

We check now if a similar switching process here is affecting the frustration of the link weight along the transient before the ensemble collapses into the final network structure. If the weight $w_{n m}$ is oscillating, we can calculate its actual frequency of oscillation $\Omega_{n m}^{\text {expt }}$ from the data, and compare it to the theoretical value, which is half the difference between the instantaneous frequencies of the linked nodes, $\Omega_{n m}^{\text {th }}:=$ $\left(\dot{\theta}_{n}-\dot{\theta}_{m}\right) / 2$. For all those values of $\sigma$ and $p_{c}$ for which the transient is especially long, we repeated the simulation of Eqs. (1) and (2) several times, each time starting from a 


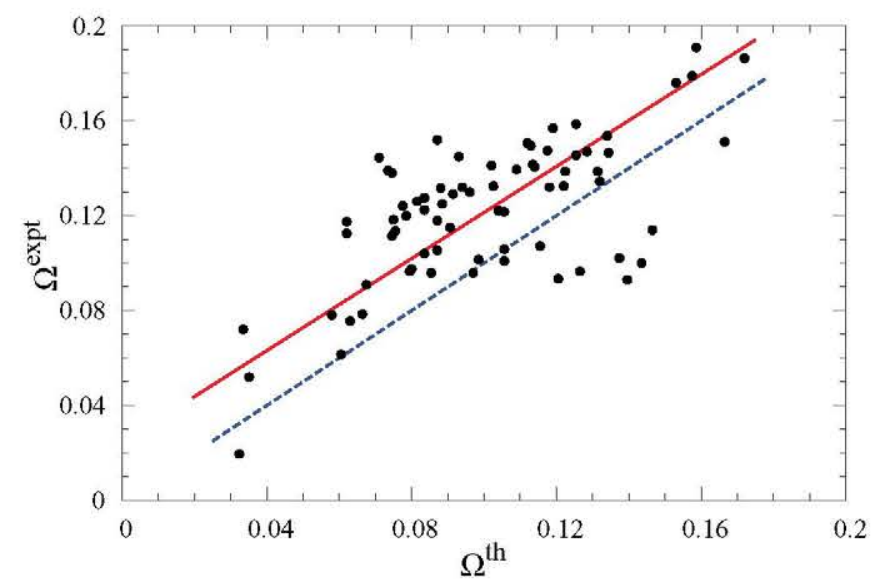

FIG. 3. (Color online) Frequency of the oscillating links in the network $\Omega^{\text {expt }}$ vs the theoretically predicted frequency $\Omega^{\text {th }}$ (see text for the definition of both quantities). When the Pearson's linear correlation coefficient is calculated, a $p$ value of $3 \times 10^{-10}$ is found, indicating that the trend is significative. The red solid line is the best fit of our data, while the blue dashed line is the theoretical prediction from Ref. [16].

different random initial condition for all $\theta_{n}(0)$ and all $w_{n m}(0)$, and measured both $\Omega_{n m}^{\text {expt }}$ and $\Omega_{n m}^{\text {th }}$. The results are shown in Fig. 3, where each point is one of the (randomly chosen) frustrated links found in those simulations. A Pearson's linear correlation coefficient test for the trend using the method of Ref. [17] yielded a best fit with a slope of 0.970 , with a Pearson coefficient of 0.662 , and a $p$ value of $3 \times 10^{-10}$, showing that the length of the transient is related to the presence of frustrated links. This represents an interesting extension of Ref. [16], where frustration was found for nodes. In the present study, each node is perfectly integrated in its cluster, and frustration instead affects the links between nodes in different communities.

In conclusion, we have shown that an initial ensemble of interacting phase oscillators can be suitably shaped into a complex and structured networked system, under the action of an adaptation mechanism that promotes (weakens) those interactions between elements that are synchronized (unsynchronized). In particular, we have characterized how the dynamical organization of the oscillators leads to modularity and assortativity features which emerge spontaneously and simultaneously. In turn, we also proved that such an emergent structure is associated with a cluster synchronization of the network. Our results can increase the understanding of the mechanisms at the basis of the connectivity and dynamical organization of some relevant cases in biology (as, e.g., brain structures), where, indeed, both partial synchronization and modular and correlated structure of the connectivities have been largely unveiled [18-20].
[1] S. Boccaletti, V. Latora, Y. Moreno, M. Chavez, and D.-U. Hwang, Phys. Rep. 424, 175 (2006).

[2] S. Fortunato, Phys. Rep. 486, 75 (2010).

[3] J. A. Almendral, R. Criado, I. Leyva, J. M. Buldú, and I. SendiñaNadal, Chaos 21, 016101 (2011).

[4] A. A. Rad, I. Sendiña-Nadal, D. Papo, M. Zanin, J. M. Buldú, F. del Pozo, and S. Boccaletti, Phys. Rev. Lett. 108, 228701 (2012).

[5] M. Girvan and M. E. J. Newman, Proc. Natl. Acad. Sci. USA 99, 7821 (2002).

[6] T. Opsahl, V. Colizza, P. Panzarasa, and J. J. Ramasco, Phys. Rev. Lett. 101, 168702 (2008).

[7] V. D. Blondel, J.-L. Guillaume, R. Lambiotte, and E. Lefebvre, J. Stat. Mech.: Theory Exp. (2008) P10008.

[8] Y. Kuramoto, Lect. Notes Phys. 39, 420 (1975).

[9] Y. Kuramoto, Chemical Oscillations, Waves, and Turbulence (Springer, New York, 1984).

[10] R. Gutiérrez, A. Amann, S. Assenza, J. Gómez-Gardeñes, V. Latora, and S. Boccaletti, Phys. Rev. Lett. 107, 234103 (2011).
[11] J. A. Acebrón, L. L. Bonilla, C. J. Pérez-Vicente, F. Ritort, and R. Spigler, Rev. Mod. Phys. 77, 137 (2005).

[12] M. E. J. Newman, Proc. Natl. Acad. Sci. USA 103, 8577 (2006).

[13] M. E. J. Newman, Phys. Rev. E 69, 066133 (2004).

[14] M. E. J. Newman, Phys. Rev. Lett. 89, 208701 (2002).

[15] R. Pastor-Satorras, A. Vázquez, and A. Vespignani, Phys. Rev. Lett. 87, 258701 (2001).

[16] D. Li, I. Leyva, J. A. Almendral, I. Sendiña-Nadal, J. M. Buldú, S. Havlin, and S. Boccaletti, Phys. Rev. Lett. 101, 168701 (2008).

[17] H. Thomas and P. Lewicki, Statistics: Methods and Applications (StatSoft, Tulsa, OK, 2006).

[18] V. M. Eguíluz, D. R. Chialvo, G. A. Cecchi, M. Baliki, and A. V. Apkarian, Phys. Rev. Lett. 94, 018102 (2005).

[19] D. S. Bassett, E. Bullmore, B. A. Verchinski, V. S. Mattay, D. R. Weinberger, and A. Meyer-Lindenberg, J. Neurosci. 28, 9239 (2008).

[20] K. E. Joyce, P. J. Laurienti, J. H. Burdette, and S. Hayasaka, PLoS ONE 5, e12200 (2010). 